\title{
Recent Developments in Minimally and Truly Non-Invasive Blood Glucose Monitoring Techniques
}

\author{
Heungjae Choi \\ School of Engineering, Cardiff University, Cardiff CF10 4JD, United Kingdom \\ ChoiH1@cardiff.ac.uk
}

\begin{abstract}
The aim of this paper is to introduce the recent research and commercial developments in minimally and noninvasive blood glucose monitoring techniques.
\end{abstract}

Keywords—Blood glucose, monitoring, non-invasive, sensor.

\section{INTRODUCTION}

Diabetes is one of the largest global health emergencies of the 21st century [1]. More than 1 in 11 adults (415 million) were found to have diabetes in 2015 and this number is rapidly rising, being estimated to reach almost 1 in 10 adults (642 million) by 2040. At least 673 billion USD in health expenditure were spent on the care of diabetes related complications, including direct and indirect costs. In the United Kingdom, $17 \%$ of the total spending by the National Health Service is estimated to be spent on diabetes in 2035/2036 [2][3]. There are significant amount of ongoing researches but there is no known cure for diabetes yet.

For people with diabetes, the most important requirement is a tight control of the blood glucose (BG) level to minimize the dwelling time in high $(>11.1 \mathrm{mmol} / \mathrm{L})$ or low $(<3.9 \mathrm{mmol} / \mathrm{L})$ BG levels. The most effective method to achieve tight control is to self-monitor the BG levels as frequent as possible. Fingerprick test by using test strips and a glucose meter is the most common and widely available method for its reasonable accuracy and precision, convenience, and its ability to provide real-time plasma glucose levels on demand. Wide range of commercial glucose meters and reagent test strips are available including Abbott's Freestyle line, Ascensia Diabetes Care's (formerly Bayer) Contour line, GlucoRX's Nexus line, LifeScan's OneTouch line, and Roche's Accu-Check line, to name a few. However, a number of factors discourage the use of this method, especially the pain due to repeated finger-prick with lancets to draw blood sample, fear of infection during the pricking process, cost of test accessories, and the invasiveness to their daily life. One of the critical disadvantages of the fingerprick test is that it is only able to provide a discrete snapshot of the continuous change of BG levels during the day. Therefore one might miss important hyperglycemic and hypoglycemic events between the discrete fingerprick tests.

To overcome this issue, continuous glucose monitoring (CGM) methods have been developed. The most common method is to have a flexible needle placed under the skin with a data transmitting device sitting on the skin, which continuously monitors interstitial fluid (ISF) glucose levels. Among several techniques, an enzyme-based electrochemical sensing method

This work was supported by the Wellcome Trust [WT096848MA]; the Ser Cymru II Fellowship[80762CU083]. is adopted for commercial products for its specificity and decent accuracy, and small footprint required. This technique has contributed in improving the quality of life of people with diabetes by reducing the required number of fingerprick tests and providing the continuous trace of fluctuation of glucose levels. With the rapid development of circuit integration technologies, CGMs have made a great leap and are becoming more and more popular. Still, the flexible subcuraneous needle has to be inserted into the skin by an invasive guide needle and therefore same disadvantages still exist regarding the invasiveness of the technique, including the pain and fear of infection and the replacement of the sensor every few weeks. Therefore the demand and expection for a truly non-invasive blood glucose monitor is growing higher and higher.

\section{RESEARCH PUBLICATIONS}

A number of minimally invasive and purely non-invasive blood glucose monitoring devices have been reported based on a wide range of interesting technologies [4]-[7]. In [8], ultrasound was facilitated to enhance the outward transport of glucose from ISF through skin to overcome the fundamental problem of obtaining sufficient amount of samples in a transdermal approach (sonophoresis). A small implantable fluorescence-based sensor combined with a body-worn data transmitter was proposed in [9] to increase the sensor lifetime up to 6 months comparing to a few weeks' lifetime of typical subcutaneous sensors. A watch-type transdermal sensor using microneedles to extract ISF glucose through skin [10], an ultrasound and multi-sensor-based ear-clip type non-invasive sensor for discrete measurement [11], a contact-lens-type noninvasive sensor measuring glucose concentration in tears [12], an occlusion spectroscopy-based sensor measuring blood flow [13], and electrochemical subcutaneous continuous monitoring sensors [14] have been reported. A sensor based on the combination of impedance ( 10 to $76 \mathrm{kHz}$ range) and multiwavelength $(850 \mathrm{~nm}, 950 \mathrm{~nm}, 1300 \mathrm{~nm})$ near infra-red (NIR) spectroscopy with a dedicated integrated circuit [15] and a technique measuring the refraction of visible laser light to overcome the signal to noise ratio issue related to NIR spectroscopy [16] were also reported. Wearable sensors capable of measuring glucose concentration through saliva and sweat [17][18], and a benchtop system capable of estimating glucose level through breath have been published [19][20].

In the RF and microwave area, the resonant and nonresonant near-field monitoring technique has been widely used for its non-invasive characteristic and high sensitivity. A broadband coplanar waveguide sensor with the combination of microfluidic system was proposed in [21] to evaluate the 
relative effective permittivity of human umbilical vein endothelial cells. An open-ended spiral-shaped microstrip line sensor was proposed for non-invasive BG measurement but with limited dataset from 5 subjects [22]. A reflection and transmission based broadband measurement techniques using microstrip line [23] and microstrip patch/slot antenna-based sensor [24] can be found, but without human clinical trial results. Millimeter-wave waveguide transmission measurement system and its clinical trial results on rats [25] have been reported using microwave techniques. The basis of these is that the complex dielectric constant changes with the presence and fluctuation of blood glucose concentration in the water/glucose system [26][27]. Microwave non-invasive blood glucose monitoring sensors based on split-ring resonators have also been reported with human clinical trial results, showing its potential as a candidate method [28][29].

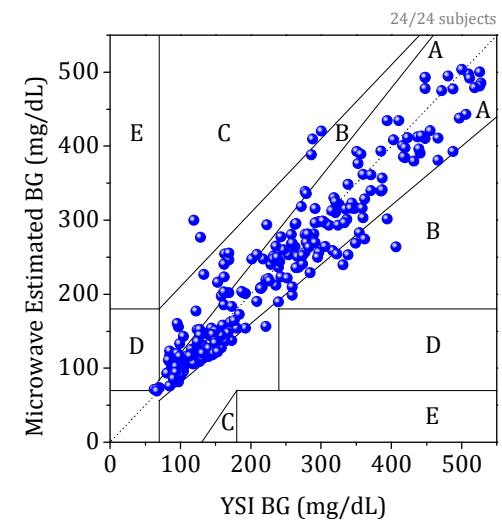

Fig. 1. Human clinical trial results of split-ring resonator sensor over 24 test subjects in Clarke Error Grid, from [29].

\section{COMMERCIAL DEVELOPMENTS}

There is no truly non-invasive and continuous blood glucose monitoring sensor availabe in the self-monitoring of BG market yet. It is extremely difficult challenge to be able to detect only a small fluctuation in BG concentration through a very lossy human tissue, with endogenic chemical interference, with physiological variation between subjects, during a normal daily life (environmental variation and movement) to name a few. On top of that, accuracy and precision requirement by the medical regulation and standard (e.g., ISO:15197:2013) is ever increasing. In its current version, ISO:15197 requires that 95\% of BG test results should meet the following key requirements:

- Within $\pm 15 \mathrm{mg} / \mathrm{dL}$ (or $0.83 \mathrm{mmol} / \mathrm{L}$ ) of laboratory results at concentrations under $100 \mathrm{mg} / \mathrm{dL}$ (or $5.6 \mathrm{mmol} / \mathrm{L}$ )

- Within $\pm 15 \%$ of laboratory results at concentrations of $100 \mathrm{mg} / \mathrm{dL}$ (or $5.6 \mathrm{mmol} / \mathrm{L}$ ) or more.

- For type 1 diabetes, 99\% of readings must fall within zones $\mathrm{A}$ and $\mathrm{B}$ of the Consensus Error Grid

However there are several candidates that provide near-noninvasive performance, and some examples are now discussed.

GlucoTrack model DF-F (by Integrity Applications) is a CE-Mark approved device, which consists of an ear clip type sensor and a main display unit. It is known to use ultrasound, electromagnetic and temperature measurements with a unique algorithm to weight each measurement and calculate the weighted average of the three readings [11]. Technically this device is truly non-invasive but in their current version only discrete readings are available and the accuracy is not as good as enzyme-based subcutaneous sensors. The ear clip must be replaced every 6 months for reliable measurements. Clinical trial results show that $97.3 \%$ of the readings fall within Zone A and $\mathrm{B}$ in the Clarke Error Grid [11].

SugarBEAT (by Nemaura Medical) is a patch type sensor, known to use the technique called reverse iontophoresis [30]. It passes a mild, non-perceptible electric current across the skin to draw a small amount of glucose molecules from the ISF into a patch placed on the skin. The recent clinical trial consisted of 81 paired data points, with a single finger prick calibration, and glucose range between $70-400 \mathrm{mg} / \mathrm{dl}$. The results indicated a mean absolute relative difference (MARD) of $14.05 \%$, and $75 \%$ of the data in Zone A, and over $98 \%$ of the data in Zones $\mathrm{A}$ and $\mathrm{B}$ of the Clarke Error grid. The patch lasts one day and needs one finger prick calibration a day. The device is aimed to be launched in 2017.

NovioSense is a small and flexible spring like device measuring less than $2 \mathrm{~cm}$ in length and $1.5 \mathrm{~mm}$ in diameter [31]. The whole device is coated with a soft hydrogel layer. The flexible form factor allows the device to bend to conform to the surface of the lower eye lid where the sensor is placed. This device uses a glucose oxidase electrochemical sensor attached to a hollow coil coated in hydrophilic gel allowing the sensor to follow the shape of the eye. It utilizes state of the art low-power and highly sensitive ASIC technology developed by the Fraunhofer Institute of Microelectronic Circuits and Systems together with NovioSense. The device can measure minute changes in glucose levels in the tears that are representative of blood glucose levels. Through animal studies, $92 \%$ of data were in Zone $\mathrm{A}+\mathrm{B}$ region of the Clarke Error Grid. The device is aimed to be launched in 2018 .

One critical challenge of measuring glucose concentration through ISF is the dispersion delay. Dispersion delay is the time for plasma glucose to disperse from blood vessel to ISF. This can vary from 5 to 45 minutes between subjects, and can even vary for a specific subject on a different day. Tear, saliva, or sweat have even longer dispersion delay than ISF, so they are not suitable for type 1 diabetes.

The Eversense Sensor is designed to be implanted subcutaneously in the upper arm [32]. No part of the sensor protrudes out of the skin surface. Encased in a biocompatible material, the sensor utilizes a patented fluorescent, glucose indicating polymer technology to measure glucose in the ISF. The measurement is then relayed to the smart transmitter. The measurement and display of glucose values is done automatically, without the need for user intervention. Senseonics' Eversense CGM has shown exceptional results in a clinical study: $8.8 \%$. The PRECISE II clinical study enrolled 90 adults with diabetes over 90 days of continuous glucose sensor wear. The study collected over 16,000 comparative data points with over 2 million glucose readings. Although simple, this device requires a surgical operation.

The FreeStyle Libre (by Abbott) is a flash glucose monitoring system which allows people to test their BG levels 
by scanning a subcutaneous sensor on their arm measuring ISF glucose rather than taking finger-prick blood glucose tests [33]. The system is able to provide graphical trends of sugar levels which provides much more data than blood glucose tests. Unlike CGMs, the Libre is not able to alert the user of high or low blood sugar until the user actively scans. However, the trendline shows whether sugar levels are heading up or down, and how quickly, which alarms the user of the onset of hypoglycemia. The FreeStyle Libre system has a proven accuracy of $11.4 \%$ MARD compared to a reference BG concentration. It is clinically proven to be accurate, stable and consistent over 14 days without the need for finger prick calibration. To be able to insert the flexible subcutaneous needle, an insertion device with a big guide needle must be used.

\section{CONCLUSION}

Diabetes prevalence is on the rapid rise worldwide and is a crisis facing $21^{\text {st }}$ century society. Therefore, the selfmonitoring blood glucose device market is exponentially growing. A variety of techniques to achieve minimally invasive and truly non-invasive blood glucose monitoring have been reviewed, including RF, microwave, mm-wave, and even optic wavelength, with highly promising results.

\section{REFERENCES}

[1] International Diabetes Federation. IDF Diabetes Atlas, 7 ed. Brussels, Belgium: International Diabetes Federation, 2015.

[2] “2015 annual report,” Diabetes UK, London, U.K., 2016.

[3] S. Wild, G. Roglic, A. Green, R. Sicree, and H. King, "Global prevalent of diabetes," Diabetes Care, vol. 27, no. 5, pp. 1047-1053, May 2004.

[4] D. C. Klonoff, "Noninvasive blood glucose monitoring," Diabetes Care, vol. 20, no. 3, pp. 433-437, Mar. 1997.

[5] O. S. Khalil, "Spectroscopic and clinical aspects of noninvasive glucose measurements," Clin. Chem., vol. 45, no. 2, pp. 165-177, Feb. 1999.

[6] S. Vaddiraju, D. J. Burgees, I. Tomazos, F. C. Jain, and F. Papadimitrakopoulos, "Technologies for continuous glucose monitoring: current problems and future promises" J. Diabetes Sci. Technol., vol. 4, no. 6, pp. 1540-1562, Nov. 2010.

[7] S. K. Vashist, "Continuous glucose monitoring systems: A review," Diagnostics, vol. 3, no. 4, pp. 385-412, Oct. 2013.

[8] J. Kost, S. Mitragotri, R. A. Gabbay, M. Pishko, and R. Langer, "Transdermal monitoring of glucose and other analytes using ultrasound," Nature Med., vol. 6, no. 3, pp. 347-350, Mar. 2000.

[9] A. DeHennis et al., "An integrated wireless fluorimeter for a long term implantable, continuous glucose monitoring system," presented at the 7th Int. Adv. Technol. Treatments for Diabetes Conf., Vienna, Austria, Feb. 5-8, 2014.

[10] F. Chowdhury and Y. Han, "Comparative performance between the former Glucowatch ${ }^{\circledR}$ and an improved non-invasive continuous glucose monitoring watch (CGM Watch)," presented at the 7th Int. Adv. Technol. Treatments for Diabetes Conf., Vienna, Austria, Feb. 5-8, 2014.

[11] A. Gal et al., "Calibration schemes of a truly non-invasive glucose monitor for variety of diabetics," presented at the 7th Int. Ad. Technol. Treatments for Diabetes Conf., Vienna, Austria, Feb. 5-8, 2014.
[12] Y. Liao, H. Hao, A. Lingley, B. Parviz, and B. Otis, "A 3-uW CMOS glucose sensor for wireless contact-lens tear glucose monitoring," IEEE J. Solid-State Circuits, vol. 47, no. 1, pp. 335-344, Jan. 2012.

[13] O. Amir et al., "Continuous noninvasive glucose monitoring technology based on occlusion spectroscopy," J. Diabetes Sci. Technol., vol. 1, no. 4, pp. 463-469, Jul. 2007.

[14] M.Schoemaker, G.Schmelzeisen-Redeker, andJ.Jager, "CGM sensor design principles for reliable and accurate glucose monitoring in the subcutaneous tissue," presented at the 7th Int. Adv. Technol. Treatments for Diabetes Conf., Vienna, Austria, Feb. 5-8, 2014.

[15] K. Song et al., "An impedance and multi-wavelength near-infrared spectroscopy IC for non-invasive blood glucose estimation," IEEE $J$. Solid-State Circuits, vol. 50, no. 4, Apr. 2015.

[16] H. Ali and F. Jaber, "Novel Approach to Non-Invasive Blood Glucose Monitoring based on Transmittance and Refraction of Visible Laser Light," IEEE Access, vol. 5, pp. 9163-9174, May 2017.

[17] T. Arakawa et al., "Mouthguard Biosensor Integrated with Wireless Module for Monitoring Human Oral Information," 2017 Int'l Conf. Electronics and Packaging (ICEP), Yamagata, Japan, 19-22 Apr. 2017.

[18] E. Cho, M. Mohammadifar, and S. Choi, "A self-powered sensor patch for glucose monitoring in sweat," 2017 IEEE $30^{\text {th }}$ Int'l Conf. Micro Electro Mechanical Systems (MEMS), Las Vegas, NV, USA, 22-26 Jan. 2017.

[19] Z. Wang et al., "Breath acetone analysis of diabetic dogs using a cavity ringdown breath analyzer," IEEE Sensors J., vol. 14, no. 4, pp. 11171123, Apr. 2014.

[20] K. Yan et al., "Design of a breath analysis system for diabetes screening and blood glucose level prediction," IEEE Trans. Biomedical Eng., vol. 61, no. 11, pp. 2787-2795, Nov. 2014.

[21] K. Grenier et al., "Integrated broadband microwave and microfluidic sensor dedicated to bioengineering," IEEE Trans. Microw. Theory Techn., vol. 57, no. 12, pp. 3246-3253, Dec. 2009.

[22] B. R. Jean, E. C. Green, and M. J. McClung, "A microwave frequency sensor for non-invasive blood-glucose measurement," in IEEE Sensors Appl. Symp., Atlanta, GA, USA, 2008, pp. 4-7.

[23] M. Hofmann, G. Fischer, R. Weigel, and D. Kissinger, "Microwavebased noninvasive concentration measurements for biomedical applications," IEEE Trans. Microw. Theory Techn., vol. 61, pp. 21952204, May 2013.

[24] T. Yilmaz, R. Foster, and Y. Hao, "Broadband tissue mimicking phantoms and a patch resonator for evaluating noninvasive monitoring of blood glucose levels," IEEE Trans. Antennas Propag., vol. 62, no. 6, pp. 3064-3075, Jun. 2014.

[25] P. H. Siegel et al., "First millimeter-wave animal in vivo measurements of L-glucose and D-glucose: further steps towards a non-invasive glucometer," in $41^{\text {st }}$ Int'l Conf. Infrared, Millimeter, and Terahertz Waves (IRMMW-THz), Copenhagen, Denmark, 25-30 Sept. 2016.

[26] J. H. Pack, C. S. Kim, B. C. Choi, and K. Y. Ham, "The correlation of the complex dielectric constant and blood glucose at low frequency," Biosens. Bioelectron., vol. 19, no. 4, pp. 321-324, Dec. 2003.

[27] A.Caduff, E.Hirt, Y.Feldman, Z.Ali, andL.Heinemann, "Firsthuman experiments with a novel non-invasive, non-optical continuous glucose monitoring system," Biosens. Bioelectron., vol. 19, no. 3, pp. 209-217, Nov. 2003.

[28] H. Choi, et al., "Design and in-vitro interference test of microwave noninvasive blood glucose monitoring sensor," IEEE Trans. Microw. Theory Techn., vol. 63, no. 10, pp. 3016-3025, Oct. 2015.

[29] H. Choi, S. Luzio, J. Beutler, and A. Porch, "Microwave noninvasive blood glucose monitoring sensor: human clinical trial results," in 2017 IEEE MTT-S International Microwave Symposium, Honolulu, HI, USA, 4-9 Jun. 2017. 